\title{
MECANISMO DE ACCION DE ANTICONCEPTIVOS ORALES: ¿CUMPLEN LOS ACO DE BAJAS DOSIS CON EL OBJ EIIVO DE INHIBIR LA OVULACION?*
}

\author{
Drs. Maritza Busquets C., Jessica Preisler ${ }^{\star *}$, Cecilia Poli ${ }^{* \star}$ \\ Servicio de Obstetricia y Ginecología, Hospital San Bernardo, Universidad de los Andes
}

${ }^{* *}$ Internas de Medicina

\section{RESUMEN}

La inhibición de la ovulación es el principal mecanismo o efecto buscando en los casos de utilización terapéutica o contraceptiva de los anticonceptivos orales (ACO). Los mecanismos de acción secundarios postfertilización tienen reparos éticos para aquellas pacientes que consideran la concepción como el comienzo del desarrollo de la persona humana. Quisimos saber en qué medida se inhibe la ovulación mediante la administración de ACO de bajas dosis, como primera aproximación al problema ético planteado por los efectos postfertilización y para analizar la eficacia terapéutica de los anticonceptivos orales. Se revisa la literatura entre los años 1985 y 2001 y se seleccionan tres trabajos comparables entre sí de los cuales se desprenden los resultados. En un período de seguimiento de tres meses, las mujeres que ingieren en forma adecuada ACO monofásicos combinados de bajas dosis que contienen $20 \mu \mathrm{g}$ de etinilestradiol, presentan ovulación demostrada hormonal y ecográficamente en $2,35 \%$ a $8,3 \%$ de las mujeres. Existe una tendencia al aumento de la actividad ovárica y de los diámetros foliculares a medida que transcurre el tiempo de observación. Si el objetivo terapéutico es la anovulación, estas cifras permiten considerar los ACO como terapias adecuadas. Frente a la utilización individual de los ACO en contracepción, las cifras expuestas permitirán abordar en qué medida actúan los mecanismos secundarios y así considerar parámetros más objetivos en el análisis de los aspectos éticos a evaluar por el médico y la paciente.

\section{PALABRAS CLAVES: Anticonceptivos de bajas dosis}

\section{SUMMARY}

Oral contraceptives (OCs) may be used for therapeutic or family planning purposes. Inhibition of ovulation is the main mechanism implicated in both instances, though post-fertilization effects described as secondary mechanism in effective contraception are ethically unacceptable for some patients who identify the start of human person at fertilization. Our concern was to establish the anovulatory power of low dose OCs in attempting to answer the ethical question and secondarily to evaluate their therapeutic efficacy. Literature search of articles published from 1985 through december 2001 using Medline concluded with 15 articles (meaningful to this theme) who met inclusion criteria: low dose monophasic preparation containing $0.20 \mathrm{mg}$ ethinylestradiol, ovulation assessed by means of hormonal and transvaginal ultrasound monitoring and the trial carried out over a period of at least three treatment cycles. Three studies are analized. We conclude that in $2.35 \%$ to $8 \%$ of women, ovulation was confirmed.This results indicate that low-dose

*Documento recibido en julio de 2002 y acepado para publicación por el Comité Editor en agosto de 2002. 
contraceptive (containing $20 \mu \mathrm{g}$ ethinylestradiol) is an effective therapeutic agent and provides the first approach in the discussion of the ethical problem related to post-fertilization effect of contraceptive.

\section{KEY WORDS: Oral contraceptives low dose}

\section{INTRODUCCION}

Los anticonceptivos orales (ACO) usados principalmente como método de planificación familiar tienen también indicaciones terapéuticas. En casos de algia pelviana secundaria a endometriosis, síndrome premenstrual, dismenorrea esencial, procesos inflamatorios pelvianos crónicos, el suprimir la ovulación es un objetivo que cumple con lograr una mejoría sintomática de estas pacientes (1-3).

En pacientes que consultan por metrorragia disfuncional o sangramiento uterino anormal, la utilización de ACO es útil en el manejo sintomático de estas patologías (4). En el síndrome de ovario poliquístico -patología hoy en día de diagnóstico frecuente- los anticonceptivos orales combinados mediante su efecto hormonal son efectivos en disminuir el hirsutismo y el acné (5).

Los anticonceptivos orales de altas dosis inicialmente comercializados en la década del 60 contenían altas dosis de estrógenos y progestágenos. Los estrógenos y los gestágenos a grandes dosis pueden inhibir por sí solos la ovulación y utilizados en conjunto, su efecto sinérgico permite disminuir la dosis de cada uno (6). La inhibición sobre el eje hipotálamo-hipófisis es dosis dependiente, a menores dosis, los niveles basales de gonadotrofinas son mayores $(7,8)$. Considerando los ACO que poseen etinilestradiol (EE2) y levonorgestrel (LNG) como progestágeno, ha habido una reducción de las dosis de ambos compuestos pasando de $50 \mu \mathrm{g}$ EE2 / $250 \mu \mathrm{g}$ LNG a las dosis actuales de $30 \mu \mathrm{g}$ EE2 / $150 \mu \mathrm{g}$ LNG. Se conserva una relación entre ambos de 1:5 que parece ser necesaria para mantener un adecuado balance en el metabolismo de carbohidratos y lípidos (9). Estos anticonceptivos que contienen dosis menores de ambos compuestos son llamados de "microdosis" y contienen actualmente $20 \mu \mathrm{g}$ EE2 y $100 \mu \mathrm{g}$ de LNG.

Los ACO tienen efecto a distintos niveles en el aparato reproductor femenino. Estos efectos son los buscados según la finalidad con la que son administrados.

En primer lugar; al ser utilizados como contraceptivos, además del efecto antiovulatorio considerado como el mecanismo principal, se describen mecanismos secundarios que consisten en los efectos sobre la migración espermática y en el endometrio. Estos mecanismos de acción secunda- rios actuarían en la medida que se produzca la ovulación, es decir, que fallara la inhibición de esta última como mecanismo principal. El efecto endometrial que pudiera alterar la capacidad de implantación del embrión puede constituirse como mecanismo anti-implantatorio. Este efecto que impide la nidación de un embrión en su primera semana del desarrollo, constituye un problema serio para aquellos que consideran la fertilización como el inicio de la persona humana. Estas últimas posturas, muchas veces compartidas por los médicos, se basan en creencias o ideales de orden religioso, cultural y/ o ético-moral. Para que los mecanismos secundarios tengan una real participación en la eficacia de los ACO como contraceptivos, una de las primeras preguntas que debe formularse es en qué porcentaje se inhibe la ovulación en la utilización de ACO. Posteriormente deberá analizarse la contribución de cada uno de los mecanismos secundarios.

En segundo lugar la utilización de estos fármacos en el arsenal terapéutico del médico, persigue inhibir la ovulación, disminuir la cantidad de sangrado, la dismenorrea, etc. Si la mujer tiene actividad sexual, aunque sea con motivos diferentes al de la planificación familiar, la administración del ACO también plantea un problema desde el punto de vista ético, ya que están en juego los mismos mecanismos descritos.

Por último, desde el sólo punto de vista terapéutico es interesante saber si con los ACO de microdosis $(0,020 \mathrm{mg}$ de etinilestradiol) estamos consiguiendo el objetivo anovulatorio deseado para el tratamiento sintomático de las pacientes.

La pregunta que nos hemos efectuado es si con la utilización de ACO de microdosis se inhibe la ovulación de manera que sea una terapia anovulatoria eficiente y que no plantee ni al médico ni a la paciente resolver un problema de orden moral. Para responderla hemos revisado trabajos clínicos experimentales que puedan ser comparables entre sí y en los cuales se investiga la supresión de la ovulación en mujeres normales que ingieren ACO de microdosis.

\section{MATERIAL Y METODO}

En esta revisión se buscó literatura desde el año 1985-2001 en diversas revistas médicas de ginecología y MEDLINE bajo los ítems "anticoncep- 
Tabla I

EFS= ESTRUCTURA FOLICULO SIMIL; LUF= FOLICULO LUTEINIZANTE SIN RUPTURA; 0,1 nmol/lt= $30 \mathrm{pg} / \mathrm{ml}$ Es; $5 \mathrm{nmol} / \mathrm{lt}=1,6 \mathrm{ng} / \mathrm{ml}$ prog. SEGUN HOOGLAND Y SKOUBY (28)

\begin{tabular}{lccc}
\hline & Diámetro folicular $(\mathrm{mm})$ & $\begin{array}{c}\text { Hormonas plasmáticas } \\
\text { Estrógenos }(\mathrm{nmol} / \mathrm{lt})\end{array}$ & Progesterona (nmol/lt) \\
\hline 1. Sin actividad & $>10$ & & \\
2. Actividad potencial & $>10$ & $<0,1$ & $<5$ \\
3. EFS sin actividad & $>13$ & $>0,1$ & $>5$ \\
4. EFS activo & $>13$ & $>0,1$ & $>5$ \\
5. LUF & $>13$, persistente & $>0,1$ & \\
6. Ovulación & $>13$, con ruptura & & \\
\hline
\end{tabular}

ción hormonal, anticoncepción oral, e "inhibición de la ovulación", "inhibition of ovulation", "oral contraceptives" y "ovulation". Se encontraron 18 trabajos (10-27) en que se analizaba la actividad ovárica -específicamente la ovulación- en la utilización de microdosis de ACO monofásicos combinados continuos de los cuales se eligieron 3 trabajos experimentales (25-27) que eran comparables entre ellos según los siguientes criterios de inclusión: 1) población semejante en cuanto a edad (18-35 años) y antecedentes ginecológicos: 2) número de ciclos analizados (5: 1 pretratamiento, 3 en tratamiento y 1 post-tratamiento); 3) el método para determinar la ovulación en todos los ciclos: ecografía transabdominal o transvaginal, y análisis hormonal de muestras de sangre (FSH, LH, $17 \beta$ estradiol y progesterona). También se consideró el hecho de que todos fuesen ACO de bajas dosis de EE2 (20 $\mu \mathrm{g}$ de etinilestradiol) asociados a levenorgestrel, desogestrel o a gestodene. Todos los estudios se llevaron a cabo sobre un total de 5 ciclos. En un primer ciclo, sin administración de los fármacos, se aseguró que las mujeres, en condiciones normales, presentaron ciclos ovulatorios. Luego se analizan 3 ciclos de tratamiento durante los cuales se administraron diariamente por 21 días, tabletas que contenían $20 \mu \mathrm{g}$ de EE2 + $100 \mu \mathrm{g}$ de LNG, $150 \mu \mathrm{g}$ de desogestrel o $75 \mu \mathrm{g}$ de gestodeno. Finalmente se analizó un último ciclo sin tratamiento. El primer ciclo de tratamiento comenzó el primer día de sangramiento menstrual y se continuó durante los 21 días consecutivos; luego de un intervalo de siete días sin tabletas se recomenzó la administración exactamente 4 semanas después de la primera píldora.

Las voluntarias consistían en un grupo de mujeres entre 18 y 35 años de edad, que cumplían con los criterios de inclusión y exclusión usuales para el uso de ACO. La actividad ovárica se determinó según la clasificación ideada por Hoogland y
Skouby (28) que se basa en los diámetros de las estructuras folículo símiles y en niveles hormonales plasmáticos en etapa secretora (Tabla I). Con estos datos se decidió hacer una tabla comparativa para ilustrar de forma didáctica los resultados.

\section{RESULTADOS}

Los resultados de los tres trabajos se resumen en la Tabla II. En el estudio de Fitzgerald et al (22) de 53 pacientes, inicialmente distribuidas al azar en cada uno de dos grupos de tratamiento, se analizan 19 mujeres en cada grupo, luego de que 12 no ovularan y 3 se retiraran durante el primer ciclo sin tratamiento. No se demuestran ovulaciones en los ciclos tratados. Se evidencia actividad folicular grado 4 y 5 en $11-21 \%$ de las mujeres. Hay una tendencia a una mayor actividad folicular durante el segundo ciclo de tratamiento. No encuentran diferencia significativa en el número de ciclos con actividad folicular entre el grupo desogestrel y gestodeno. Los autores dan los resultados en porcentajes de mujeres sin detallar el número ni el ciclo de tratamiento en que ocurrió la actividad ovárica. El estudio de Spona et al (23) se realiza con 71 ciclos de tratamiento en 23 mujeres que completan el estudio. Tampoco se evidencian ovulaciones en estos ciclos. Los autores muestran indistintamente datos de ciclos y de mujeres. En $32,4 \%$ de los ciclos de tratamiento se objetiva actividad folicular grado 4 y 5 . La actividad residual se manifiesta principalmente como actividad tipo 4 (folículos mayores de $13 \mathrm{~mm}$ con secreción estrogénica adecuada pero niveles no ovulatorios de progesterona). En el primer ciclo de tratamiento 2 de 24 ciclos presentan actividad tipo 4; en el segundo y tercer ciclo en que se evidencia actividad residual (4 y 5) son un 50\% (12 de 24 ciclos) y 39\% (9 de 23 ciclos) respectivamente. Hay entre estos últimos, una mujer que presenta en el segundo y tercer ciclo una actividad tipo 5 catalogada como 
Tabla II

FITZGERALD ET AL (25) COMPARA DOS GRUPOS QUE VARIAN EN EL PROGESTAGENO UTILIZADO, DESOGESTREL Y GESTODENO. EN EL TRABAJO DE FITZGERALD, LOS RESULTADOS ESTAN DADOS EN PORCENTAJES DE MUJERES A DIFERENCIA DE CONEY (27) Y SPONA (26), DONDE SE EXPONEN EN PORCENTAJES DE CICLOS

\begin{tabular}{|c|c|c|c|c|}
\hline Referencia & $n$ & $\begin{array}{l}\text { Edad } \\
\text { (años) }\end{array}$ & $\begin{array}{c}\text { Desarrollo } \\
\text { folicular } 4 \text { a } 5\end{array}$ & Ovulación \\
\hline $\begin{array}{l}\text { Fitzgerald (1994): } \\
20 \mu \mathrm{g} \mathrm{EE} 2+75 \mu \mathrm{g} \text { gestodeno o } \\
150 \mu \mathrm{g} \text { desogestrel }\end{array}$ & $53(38)^{*}$ & $18-35$ & $\begin{array}{c}11-21 \% \\
\text { de mujeres tratadas }\end{array}$ & No hay \\
\hline $\begin{array}{l}\text { Spona (1996): } \\
20 \mu \mathrm{g} \text { EE2 + } 100 \mu \mathrm{g} \text { levonorgestrel }\end{array}$ & $24(23)^{*}$ & $20-34$ & $\begin{array}{c}32,4 \% \\
\text { de ciclos tratados }\end{array}$ & No hay \\
\hline $\begin{array}{l}\text { Coney (1999): } \\
20 \mu \mathrm{g} \text { EE2 + } 100 \mu \mathrm{g} \text { levonorgestrel }\end{array}$ & $26(24)^{*}$ & $<35$ & $\begin{array}{c}41,1 \% \\
\text { de ciclos tratados }\end{array}$ & $\begin{array}{c}2,7 \% \\
\text { de ciclos tratados }\end{array}$ \\
\hline
\end{tabular}

*Número de pacientes que terminaron el estudio.

LUF (Lutein Unrupture Follicle). Los diámetros foliculares son mayores en el segundo y tercer ciclo de tratamiento en comparación con el primero. No está descrito qué porcentaje de los ciclos que presentan actividad ovárica pertenecen o no a la misma mujer. La supresión de los diámetros foliculares es también más manifiesta en el primer ciclo que en el segundo y tercer ciclo de tratamiento, los autores suponen que el hecho se corrobora con la mayor actividad folicular observada en esos ciclos (Figuras 1 y 2).

Coney et al (24), a diferencia de los 2 trabajos anteriores, distingue entre ciclos de tratamiento y mujeres. En el $62 \%$ de 73 ciclos de tratamiento y en $81 \%$ de 26 mujeres se observan diámetros foliculares mayores de $10 \mathrm{~mm}$ lo cual se clasifica como actividad ovárica potencial. Esto se asocia a niveles

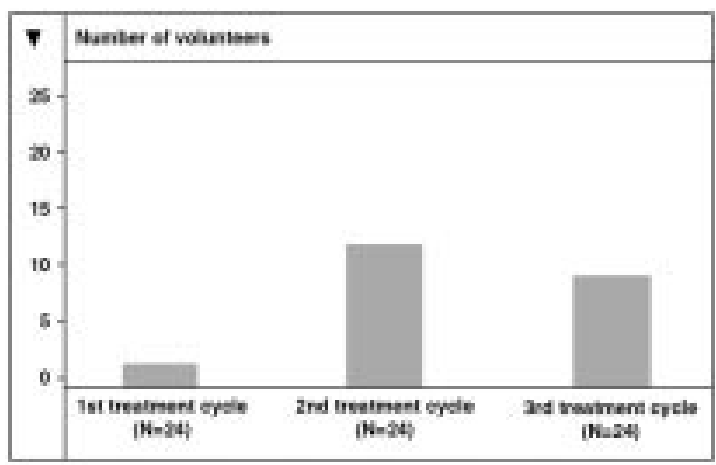

Figura 1. Número de voluntarias para las cuales se registró actividad 4 y 5 durante los tres ciclos de tratamiento con $100 \mu \mathrm{g}$ LNG más $20 \mu \mathrm{g}$ de EE2 (tomado de Spona et al. Inhibition of ovulation by an oral contraceptive containing $100 \mu \mathrm{g}$ levonorgestrel in combination with $20 \mu \mathrm{g}$ ethinylestradiol. Contraception 1996; 54: 299-304). de progesterona bajos pero a niveles fluctuantes de estradiol. En $38,4 \%$ de los ciclos hay actividad folicular tipo 4 , en $2,7 \%$ (dos mujeres) tipo 5 y en $2,7 \%$ (otras dos mujeres) hay ovulación (Tabla III). Según el ciclo de tratamiento de las mujeres de la serie, los diámetros foliculares van aumentando. Coney demuestra ovulación con folículos de 36 y $18 \mathrm{~mm}$ en dos mujeres, en el tercer ciclo de tratamiento, lo que significa un $8 \%$ de las mujeres de su serie.

En todos los trabajos se estudiaron las poblaciones, según lo enunciado en los criterios de inclusión. Sin embargo, los resultados en cuanto a número de ovulaciones, que pueden ser referidos a ciclos o a mujeres no están formulados de igual manera entre los tres trabajos. Dos de los trabajos no refieren los resultados diferenciados según ci-

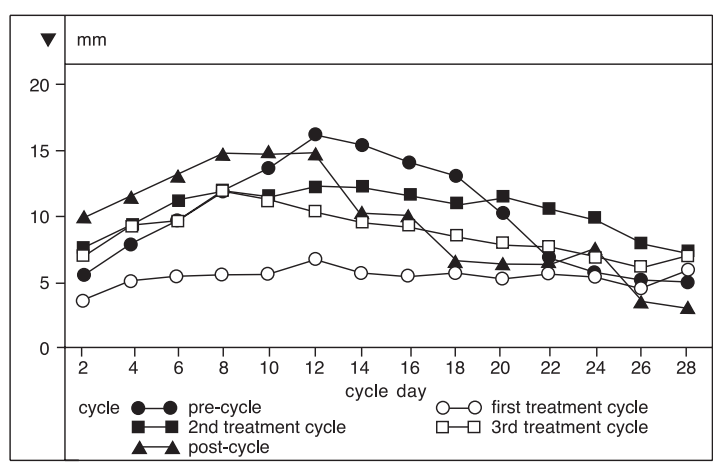

Figura 2. Media de diámetros de estructuras folículo-símiles. Antes del tratamiento, durante, y luego del tratamiento con $100 \mu \mathrm{g}$ de LNG más $20 \mu \mathrm{g}$ de EE2 (tomado de Spona et al: Inhbition of ovulation by an oral contraceptive containing $100 \mu \mathrm{g}$ levonorgestrel in combination with $20 \mu \mathrm{g}$ ethinylestradiol. Contraception 1996; 54: 299-304). 
Tabla III

\section{GRADO DE ACTIVIDAD OVARICA POR CICLOS DE TRATAMIENTO CON $20 \mu \mathrm{g}$ DE EE2 MAS $100 \mu \mathrm{g}$ DE LNG*}

\begin{tabular}{lcc}
\hline Grado & $n$ de ciclos & \% de ciclos \\
\hline 1 & 28 & 38,4 \\
2 & 12 & 16,2 \\
3 & 1 & 1,4 \\
4 & 28 & 38,4 \\
5 & 2 & 2,7 \\
6 & 2 & 2,7 \\
\hline
\end{tabular}

*Coney P, DelConte A: The effects on ovarian activity of a monofasic oral contraceptive with $100 \mu \mathrm{g}$ levonorgestrel and $20 \mu \mathrm{g}$ ethinylestradiol. Am J Obstet Gynecol 1999; 181: S53-58.

clos y según mujeres, por lo tanto no es posible tomar el total de mujeres y de ciclos de las tres series para recalcular todos los parámetros. Del total de mujeres de las tres series, que terminaron el seguimiento un $2,35 \%$ (dos mujeres de 85 presentaron ovulación pesquisada hormonal y ecográficamente).

\section{DISCUSION}

Durante la administración de ACO secuenciales de dosis bajas, la actividad ovárica no se inhibe completamente. Grimes (14) en un estudio clínico experimental describe ovulaciones en usuarias de ACO que contienen $0,35 \mathrm{mg}$ de estradiol durante los ciclos 5 y 6 de tratamiento, siendo el porcentaje de ovulación en este grupo tres veces mayor que en el grupo de ACO trifásicos que contienen 0,50 $\mathrm{mg}$ de etinilestradiol. Teichmann (15) en un estudio de diseño semejante a los que hemos analizado, objetiva actividad hormonal folicular correspondientes a grados 4 y 5 en 17 ciclos de los 39 que estudia lo que equivale a un $43 \%$ de los ciclos. No realiza seguimiento folicular en todas ellas lo que no permite saber el grado de desarrollo folicular y si hay o no ovulaciones. En los trabajos analizados, un 11 a $20 \%$ de las mujeres presentarían actividad folicular sin llegar a ovular (Fitzgerald) y hasta un $81 \%$ de las mujeres presentaron folículos de más de 10 $\mathrm{mm}$ (Coney). Se observa que los diámetros foliculares van progresivamente aumentando durante los ciclos de tratamiento, al igual que la actividad ovárica que también aumenta progresivamente hacia el tercer ciclo de tratamiento. En los trabajos de Fitzgerald y de Spona la actividad ovárica en el tercer ciclo baja con respecto al segundo, sin embargo en ambos casos la actividad ovárica en el tercer ciclo es más elevada que en el primer ciclo. Coney confirma el aumento sucesivo de los diámetros foliculares durante los ciclos de tratamiento. En otros trabajos también se ha corroborado la tendencia al aumento de la actividad hormonal folicular en segundo y tercer ciclo de tratamiento (15). Una primera conclusión es que el efecto de los ACO de bajas dosis varía durante el tiempo de exposición y que al revés de lo que pudiera suponerse en el sentido de que a más tiempo de exposición mayor inhibición del eje, esto no sucede así en la práctica. En todo caso, el presentar goteo intermenstrual, más frecuente en el primer ciclo según Fitzgerald, no es indicativo de mayor o menor actividad ovárica.

Ya que dos de los trabajos no refieren los resultados diferenciados según ciclos y según mujeres, no nos es posible tomar todas las mujeres o todos los ciclos como universo para todos los parámetros. Con los datos disponibles, y ya que no hay ovulaciones descritas en dos trabajos, sólo es posible deducir el porcentaje de mujeres que terminaron el seguimiento y que presentaron ovulación pesquisada hormonal y ecográficamente y que es de un $2,35 \%$ (dos mujeres de 85 ) del número total de mujeres que terminaron el seguimiento.

El fenómeno de escape ovulatorio ("breakthrough ovulation") que ha sido ampliamente documentado y analizado (29) plantea una disminución de la eficiencia contraceptiva en el uso poblacional de los ACO con respecto a la eficiencia medida en condiciones ideales. Esto es también esperable para los ACO de bajas dosis y el hecho de que se evidencie escape en condiciones ideales hace suponer que también habrá escape en el uso de los compuestos que nos atañen.

Aunque el perfil hormonal en fase lútea se utiliza para detectar la ocurrencia de ovulación, la ecografía transvaginal es actualmente el medio más utilizado en el diagnóstico de ovulación. La ecografía transvaginal permite también identificar la ocurrencia de LUF, en que habiendo desarrollo folicular adecuado y producción de progesterona adecuada no hay ovulación ni liberación del oocito para ser fecundado. No se describen ovulaciones en los trabajos de Spona y Fitzgerald. Coney demuestra ovulación en dos mujeres, lo que significa un $8 \%$ de las mujeres de su serie. No refiere en qué ciclos de tratamiento ocurrieron las ovulaciones. En el trabajo de Spona una misma paciente presenta LUF en los ciclos segundo y tercero de tratamiento. Esto significa un 4\% aproximadamente de las mujeres de su serie equivalente a un $2,8 \%$ de los ciclos. Coney encuentra un $2,7 \%$ de LUF entre los 
ciclos, lo cual corresponde a un $8 \%$ aproximadamente (dos casos) de las mujeres de su serie.

El concepto de LUF es actualmente discutido y el diagnóstico basado en el monitoreo ecográfico es difícil ya que los signos ecográficos no son siempre claros $(30,31)$. Se lo ha postulado como causa de infertilidad, pero también ha sido descrito en $4,5 \%$ de mujeres en edad reproductiva sin problemas de fertilidad por lo que pudiera ser considerado como una variante biológica (32). La presencia de LUF podría ser la traducción de un funcionamiento ovárico relativamente normal, y su ocurrencia en un ciclo particular, un fenómeno aislado que más bien apoya un contexto de funcionamiento ovárico normal. La ovulación es posible en mujeres usuarias de ACO como bien lo confirma la serie de Coney en que un $8 \%$ de las mujeres presentaron ovulación durante los tres primeros ciclos de ingesta de ACO en condiciones ideales. El hecho de objetivar LUF entre las usuarias de ACO viene a confirmar el hecho de que la actividad folicular no está inhibida en estas mujeres. Analizados individualmente, en el trabajo de Spona un $4 \%$ de las mujeres presenta LUF y en el de Coney un $16 \%$ de las mujeres presentaron ovulación o LUF. Fitgerald no describe ni LUF ni ovulaciones, el uso de desogestrel o gestodeno es lo único que lo diferencia de los otros dos esquemas que utilizan levonorgestrel. Si esto último es la causa de una mayor inhibición del eje es un factor que no será analizado en el presente trabajo.

No fue posible saber en qué medida los ciclos con actividad ovárica corresponden a las mismas o a distintas pacientes, en ninguno de los trabajos. Tampoco es posible saber si las pacientes que presentaron ovulación tenían actividad folicular (tipo 4) en los otros ciclos. Esto permitiría determinar si hay un grupo o tipo de pacientes en quienes el ACO no inhibe suficientemente el eje. Dado que en el trabajo de Spona la misma mujer presenta dos veces LUF pudiera pensarse que existe alguna "predisposición" de ciertas mujeres a mantener actividad ovárica bajo ACO. Sin embargo, en el trabajo de Coney las mujeres que presentan LUF son distintas y tampoco son las mismas que presentan ovulación.

Toda vez que la ovulación debe ser evitada para paliar síntomas o tratar patología de base en la mujer, el bajo porcentaje de ciclos ovulatorios en régimen con ACO de bajas dosis, hacen de éstos una arma terapéutica efectiva. La actividad folicular residual hasta de un $81 \%$ en la serie de Spona que probablemente mantiene niveles estrogénicos significativos pudiera dar cuenta de fracasos de tratamiento en patología que responde a estrógenos como por ejemplo la endometriosis. Frente al caso individual de una paciente con actividad sexual, entre un mínimo de 2,35\% y un máximo de $8 \%$ de las mujeres ovulan dentro de los tres primeros meses de uso de ACO de bajas dosis, en condiciones ideales. En caso de utilización prolongada, y en condiciones reales de utilización de contracepción, existe la posibilidad teórica de ovulaciones que ocurren después de los seis meses de tratamiento pero se ignora el porcentaje de mujeres en que ocurre.

Resolver si la utilización del ACO constituye eventualmente- un problema de orden moral para aquellos médicos y pacientes que tienen reparos a mecanismos de acción post-fertilización, como el antiimplantatorio, pasa por contestar en qué medida actúan los otros mecanismos secundarios que impiden el desarrollo de un embarazo. Esta última pregunta -cuya respuesta no es el objetivo de esta revisión- debiera ser analizada en base a trabajos que además de contar con casuística adecuada, analicen qué ocurre con el moco y el endometrio cuando se ha desencadenado la ovulación, no obstante la administración de ACO de bajas dosis.

El gran avance tecnológico y la gran disponibilidad de medicamentos así como de terapias y de formas de control o planificación familiar se ofrecen a una población de mujeres o pacientes que tienen posturas antropológicas indefinidas 0 irrelevantes para el tema del comienzo de la vida humana y también a una población con posturas particulares y claramente definidas. Este último grupo de pacientes exige frecuentemente información respecto del tema, y el médico independientemente de su opinión personal, debe dar siempre información veraz. Nos parece que estas cifras son importantes para el manejo de la información en nuestra relación con la paciente y para la eventual discusión ética actual sobre el tema.

\section{BIBLIOGRAFIA}

1. Cohen J: Les dysménorrhées. En: Chapron C, Benhamou D, Belaisch-Allart J, Dubuisson JB (eds) La douleur en Gynécologie. Paris: Arnette Blackwell SA 1997; 131-64.

2. Tamborini A: Les syndromes prémenstruals. En: Chapron C, Benhamou D, Belaisch-Allart J, Dubuisson JB (eds). La douleur en Gynécologie. Paris: Arnette Blackwell SA, 1997; 107-24.

3. Rapkin AJ: Pelvic pain and dysmenorrhea. En: Berek JS, Adashi EY, Hillard PA (eds). Novak's Gynecology. Baltimore: Williams \& Wilkins, 1996; 399-428.

4. Munro MG: Medical management of abnormal uterine bleeding. Obstet Gynecol Clin North Am 2000; 27: 287-304. 
5. Goldzieher JW: Polycystic ovarian disease. Fertil Steril 1981; 35: 371-94.

6. Stubblefield PG: Family planning. En: Berek JS, Adashi EY, Hillard PA (eds). Novak's Gynecology. Baltimore: Williams \& Wilkins 1996; 227-78.

7. Landgren BM: Mechanism of action of gestagens. Int J Gynaecol Obstet 1990; 32: 95-110.

8. Gaspard UJ, Dubois M, Gillain D, Franchimont P, Duvivier J: Ovarian function is effectively inhibited by a low dose triphasic oral contraceptive containing ethinyl estradiol and levonorgestre. Contraception 1984; 29: 305-18.

9. Arnt IV, Ferrari A, Sartoretto JN, Woutersz TB: Lowdose combination oral contraceptives: a controlled clinical study of three different norgestrel-ethinyl estradiol ratios. Fertil Steril 1997; 28: 549-53.

10. Spona J, Feichtinger W, Kindermann C, Moore C, Mellinger U, Walter F, Graser T: Modulation of ovarian function by an oral contraceptive containing $30 \mu \mathrm{g}$ ethinyl estradiol in combination with $2.00 \mathrm{mg}$ dienogest. Contraception 1997; 56: 185-91.

11. Rice CF, Killick SR, Dieben T, Coelingh Bennink H: A comparison of the inhibition of ovulation achieved by desogestrel 75 micrograms and levonorgestrel 30 micrograms daily. Hum Reprod 1999; 14: 982-5.

12. Thomas K, Vankrieken L: Inhibition of ovulation by lowdose monophasic contraceptive containing gestodene. Am J Obstet Gynecol 1990; 163(4 Pt 2): 1404-10.

13. Archer DF, Maheux R, Del Conte A, O'Bren FB: A new low-dose monophasic combination oral contraceptive (Alesse) with levonorgestrel 100 micrograms and ethinyl estradiol 20 micrograms. North American Levonorgestrel Study Group (NALSG). Contraception 1997; 55: 139-44.

14. Grimes D, Godwin A, Rubin A, Smith J, Lacarra M: Ovulation and follicular development associated with three low-dose oral contraceptives: a randomized controlled trial. Obstet Gynecol 1994; 83: 29-34.

15. Teichmann A, Martens H, Bordasch C, Petersen G, Lorkowski G: The effects of a new low-dose combined oral contraceptive containing levonorgestrel on ovarian activity. Eur J Contracept Reprod Health Care 1996; 1: 245-56.

16. Killick S, Eyong E, Elstein M: Ovarian follicular development in oral contraceptive cycles. Fertil Steril 1987; 48: 409-13.

17. Spona J, Lachnit-Fixson U, Dusterberg B, Dobianer $\mathrm{K}$ : Inhibition of ovulation by a triphasic gestodenecontaining oral contraceptive. Adv Contracept 1993; 9: $187-94$.

18. Crosignani PG, Testa G, Vegetti W, Parazzini F: Ovarian activity during regular oral contraceptive use. Contraception 1996; 54: 271-3.
19. Sullivan H, Furniss $H$, Spona J, Elstein M: Effect of 21 day and 24 day oral contraceptive regimens containing gestodene $(60 \mu \mathrm{g})$ and ethinylestradiol $(15 \mu \mathrm{g})$ on ovarian activity. Fertil Steril 1999; 72: 115-20.

20. Rosenbaum P, Schmidt $W$ et al: Inhibition of ovulation by a novel progestogen (drospirenone) alone or in combination with wthinylestradiol. Eur J Contracept Reprod Health Care 2000; 5: 16-54.

21. Lete I, Morales P: Inhibition of follicular growth by two different oral contraceptives (monophasic and trphasic) containing ethinylestradiol and gestodene. Eur J Contracept Reprod Health Care 1997; 2: 187-91.

22. Spona J, Huber J: Efficacy of low-dose oral contraceptives containing levonorgestrel, gestodene and cyproterone acetate. Gynecol Obstet Invest 1987; 23 : 184-93.

23. Wenzl R, Bennink HC, van Beek A, Spona J, Huber $\mathrm{J}$ : Ovulation inhibition with a combined oral contraceptive containing $1 \mathrm{mg}$ micronized 17 beta-estradiol. Fertil Steril 1993; 60: 616-9.

24. Teichmann A, Brill K, Albring M, Schnitker J, Wojtinek $P$, Kustra $E$ : The influence of the dose of ethinylestradiol in oral contraceptive on follicle growth. Gynecol Endocrinol 1995; 9: 299-305.

25. Fitzgerald $C$ et al: $A$ comparison of the effects of two monophasic low dose oral contraceptives on the inhibition of ovulation. Advances in contraception 1994; 10: 5-18.

26. Spona J, Feichtinger W, Kinderman $\mathrm{Ch}$ et al: Inhibition of ovulation by an oral contraceptive containing $100 \mu \mathrm{g}$ levonorgestrel in combination with $20 \mu \mathrm{g}$ ethinylestradiol. Contraception 1996; 54: 299-304.

27. Coney $\mathrm{P}$, DelConte A: The effects on ovarian activity of a mnofasisc oral contraceptive with $100 \mu \mathrm{g}$ levonorgestrel and $20 \mu \mathrm{g}$ ethinylestradiol. Am J Obstet Gynecol 1999; 181: S53-58.

28. Hoogland HJ, Skouby SO: Ultrasound evaluation of ovarian activity under oral contraceptive. Contraception 1993; 47: 583-90.

29. Larimore W, Stanford J: Postfertilization effects of the oral contraceptives and their relationship to informed consent. Arch Fam Med 2000; 9: 126-33.

30. Speroff L, Glass RH, Kase NG: Female infertility. En: Speroff L, Glass RH, Kase NG (eds). Clinical Gynecologic Endocrinology and Infertility. Baltimore: Lippincott Williams \& Wilkins 1999; 1014-42.

31. Crisosto C, Cheviakoff S: Infertilidad Conyugal. En: Pérez-Sánchez A (ed). Ginecología. Santiago, Chile: Edit Mediterráneo 1995; 381-97.

32. Kerin JF, Kirby C, Morris D, McEvoy M, Ward B, Cox LW: Incidence of the luteinized unruptured follicle phenomenon in cycling women. Fertil Steril 1983; 40: 620- 